\title{
Triatoma brasiliensis (Neiva,1911) (Hemiptera, Reduviidae): Observações Sobre seu Comportamento em Relação à Fonte Alimentar em Galinheiro Experimental
}

\author{
Triatoma brasiliensis Neiva, 1911 (Hemiptera, Reduviidae): Observations \\ on Dynamics of Feeding Behavior in an Artificial Ecotope
}

\author{
Vera Lúcia C. C. Rodrigues' ; Antenor do N. Ferraz-Filho' \\ George K. Ishihata' \& Eduardo Olavo da R. e Silva'
}

RODRIGUES, V. L. C. C.; FERRAZ-FILHO, A. N.; ISHIHATA, G. K. \& SILVA, E. O. R. Triatoma brasiliensis Neiva, 1911 (Heraiptera, Reduviidae): Observations on Dynamics of Feeding Behavior in an; Artificial Ecotope. Cad. Saúde Públ., Rio de Janeiro, 11 (2): 266-270, Apr/Jun, 1995.

In order to study the behavior of triatomine bugs under artificial ecotopic conditions, an experimental, double-walled poultry coop was built. Its inside walls were made of sun-dried clay blocks which could be broken apart for better access to the insects hiding in their crevices. The coop was subdivided into two main areas of different sizes using wire netting and a wall made of perforated bricks. The chickens, the only food source available for the triatomines, were kept in the larger, rectangular area, further subdivided diagonally. The triatomines were released in the smaller area and were allowed to migrate to the larger one though the holes in the bricks. Information about migration, survival, and hiding places of fourth-stage nymphs of Triatoma brasiliensis was thus gathered. At 30-day intervals, over a period of six months, the inner walls of the house were taken apart and inspected. The nymphs were marked with different colors according to the month and place of capture. It was found that $88.5 \%$ of the nymphs were captured near the roost and found engorged. After having taken their first blood meal, they would remain in the vicinity of their food source. The results obtained are considered epidemiologically relevant, and particularly so as pertaining to the control of the vectors of Chagas' disease.

Key words: Triatoma brasiliensis; Triatominae; Chagas' Disease

\section{INTRODUÇÃO}

A espécie Triatoma brasiliensis (Neiva, 1911) em seu habitat natural nas zonas semiáridas e de clima quente, domínio das caatingas (Forattini, 1980). Sua distribuição se estende pelos Estados: Piauí, Ceará, Rio Grande do Norte, Paraíba, Pernambuco, Alagoas, Bahia, Goiás e Minas Gerais (Lent \& Wygodzinsky, 1979). É uma espécie ativa, deslocando-se com rapidez nos seus esconderijos naturais, onde é

\footnotetext{
'Superintendência de Controle de Endemias, Secretaria de Estado da Saúde. Rua Afonso Pessini, 86, Caixa Postal 192, Mogi Guaçu, SP, 13840-000, Brasil.
}

encontrada em locais, os mais diversos. Os ninhos de galinhas e locas de mocós são por ele freqüentados e lhes servem de abrigo (Lucena, 1958). Lucena (1960), considera o Triatoma brasiliensis como espécie adaptada ao domicílio no nordeste. Alencar (1987), observou no Ceará que a espécie habita todos tipos de ecótopos domésticos e peridomésticos, como também é largamente encontrada em ecótopos silvestres (locas de pedras), devido a sua ampla valência ecológica (espécie euritópica). Em condições de laboratório apresenta elevada resistência ao jejum, como foi observado por Costa \& Perondini (1973).

Em vista da importância da espécie, sobretudo no nordeste, realizamos um estudo a respeito 
do deslocamento abrigo e permanência ou não junto à fonte alimentar (aves). Sendo assim o trabalho mostra os resultados alcançados, considerados de interesse para a epidemiologia e controle dos vetores da doença de Chagas.

\section{METODOLOGIA}

Estudamos o deslocamento e acomodação das ninfas do $4^{\circ}$ estádio de Triatoma brasiliensis em um galinheiro experimental $(330 \times 230 \mathrm{~cm})$, com paredes formadas por blocos de barro desmontáveis. O galinheiro foi hipoteticamente subdividido em 4 áreas, como mostra a Figura 1. As áreas I e II abrangiam os locais onde as galinhas se deslocavam, alimentavam e repousavam (poleiro). A área de separação era fechada por tela de malha fina, na parte superior, e tijolos furados na parte inferior (III) permitiam livremente a passagem das ninfas do Triatoma brasiliensis da área IV, onde eram soltas as ninfas nos dias determinados. A soltura era realizada ao cair da noite como meio natural de proteção imediata contra a predação por parte das aves confinadas. As temperaturas (máxima e mínima) e a umidade no interior do galinheiro foram registradas diariamente no decorrer do experimento.

As paredes do galinheiro foram desmontadas e minuciosamente pesquisadas mensalmente, durante seis meses (março a agosto de 1992). As ninfas encontradas eram marcadas com diferentes cores de tintas usadas em aeromodelismo, de acordo com o respectivo mês da leitura e sua posição nas áreas consideradas, procedimento assemelhado ao utilizado por Mac Cord et al. (1983a). A seguir recolocadas no local do encontro, para observação futura de possível deslocamento.

De início, foram soltas 60 ninfas em jejum. A cada leitura eram repostas na área N, o número de ninfas necessário para manter a quantia inicial (60), devido à morte, desaparecimento (provável ingestão pelas galinhas) e aquelas poucas que atingiram a fase alada, para evitar posturas que iriam dificultar as leituras posteriores. Desse modo foi mantido constante o número de triatomíneos no interior do galinheiro.

FIGURA 1. Subdivisão do Galinheiro Experimental. Sucen, Mogi Guaçu, 1992

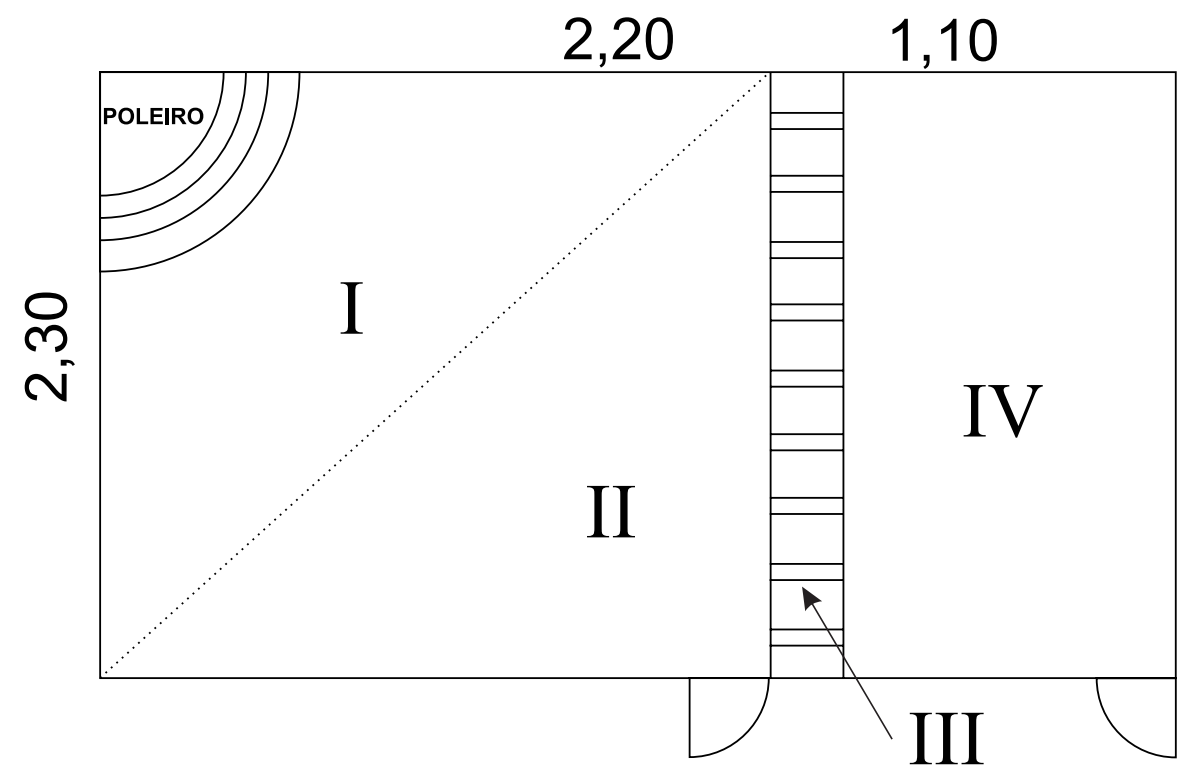

Área I - Área de Repouso da Fonte Alimentar (Poleiro)

Área II - Área de Alimentação das Aves

Área III - Barreira de Transposição entre o Local da Soltura e a Fonte de Alimentação (Tijolos Furados e Tela)

Área IV - Área de Soltura das Ninfas 
No que se refere às aves confinadas no galinheiro para a alimentação dos "barbeiros", deve-se frisar que as mesmas eram permanentemente vistoriadas para substituição em caso de morte e para mantê-las saudáveis (alimento e água).

Os números de exemplares encontrados em cada uma das leituras e sua distribuição no galinheiro foram tabulados e estão sendo apresentados na sua totalidade em tabelas e num gráfico demonstrativo de distribuição dos mesmos no terreno.

\section{APRESENTAÇÃO DOS RESULTADOS}

Os resultados alcançados no decorrer das seis leituras realizadas são mostrados na Tabela 1, onde se observa que $88,5 \%$ das ninfas engorgitadas foram encontradas nas proximidades do local de repouso da fonte alimentar (poleiro). As poucas ninfas não alimentadas se distribuíram indiferente por todas as áreas e representaram apenas $11,5 \%$ do total. A distribuição das ninfas no terreno, como mostra a Figura 2, se concentra nas proximidades do local de repouso das aves

TABELA 1. Triatoma brasiliensis - Situação das Ninfas Encontradas Vivas em Relação ao Alimento. Sucen, Mogi Guaçu, 1992

\begin{tabular}{lccc}
\hline \hline & $\begin{array}{r}\text { Posicionamento } \\
\text { em Relação } \\
\text { ao Alimento }\end{array}$ & & \\
Situação & Área I & Outras Áreas & Total \\
Alimentar & $177(88,5 \%)$ & - & $177(88,5 \%)$ \\
\hline Alimentadas & $013(06,5 \%)$ & $010(05,0 \%)$ & $023(11,5 \%)$ \\
Não Alimentadas & 190 & 10 & 200 \\
\hline Total & & & \\
\hline \hline
\end{tabular}

FIGURA 2. Gráfico Espacial da Distribuição Total das Ninfas Encontradas na Área I do Galinheiro Experimental. Sucen, Mogi Guaçu, 1992

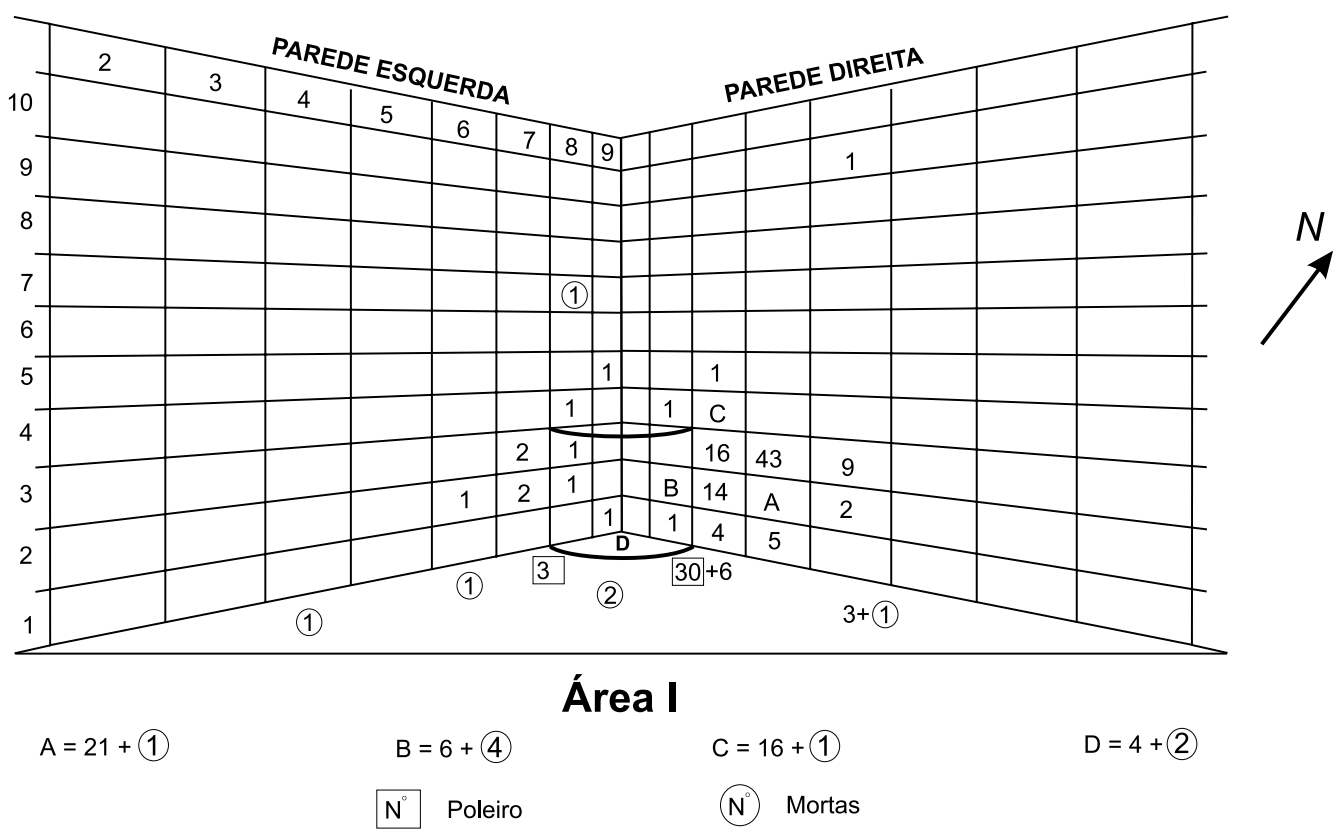


(poleiro). No que se refere à altura do encontro, observa-se sua presença na parte mais baixa das paredes, e mais acentuada, ainda, foi a presença na parede mais atingida pelos raios solares (parede direita). Esta parede, pela sua posição, era a que recebia mais sol e, portanto, mais seca e quente do que as demais, reforçado pelo período em que foi realizado o experimento, o menos chuvoso da época do ano na região. As médias mensais de temperatura e umidade relativa observadas no decurso dos seis meses, são as seguintes (Tabela 2):

TABELA 2. Médias Mensais de Temperatura e Umidade Relativa. Sucen, Mogi Guaçu, 1992

\begin{tabular}{lcccc}
\hline \hline & \multicolumn{2}{c}{ Temperatura Média ${ }^{\circ} \mathrm{C}$} & \multicolumn{2}{c}{ Umidade Relativa } \\
\cline { 2 - 5 } Mếdia $(\%)$ \\
\hline Março & Máxima & Mínima & Máxima & Mínima \\
Abril & 22,7 & 18,0 & 91,00 & 69,00 \\
Maio & 21,5 & 17,3 & 86,50 & 60,70 \\
Junho & 21,2 & 15,6 & 92,70 & 68,10 \\
Julho & 20,8 & 14,0 & 94,30 & 59,00 \\
Agosto & 20,9 & 13,0 & 93,40 & 51,50 \\
\hline Total & 22,2 & 15,3 & 88,50 & 52,60 \\
\hline \hline
\end{tabular}

Na Tabela 3 podemos podemos observar a situação alimentar das ninfas por área e se encontradas vivas ou mortas. Novamente se observa o maior encontro de ninfas vivas na área I e uma distribuição menos nítida das mortas.

TABELA 3. Triatoma brasiliensis - Situação Alimentar da Totalidade das Ninfas Encontradas em Cada Área. Sucen, Mogi Guaçu, 1992

\begin{tabular}{lcccc}
\hline \hline & \multicolumn{2}{c}{ Total de Ninfas Encontradas } & \multicolumn{2}{c}{ Situação Alimentar } \\
\cline { 2 - 5 } Área & Vivas & Mortas & Alimentadas & N/A \\
\hline I & 190 & 20 & 177 & 13 \\
II & - & - & - & - \\
III & 08 & 07 & - & 08 \\
IV & 02 & 06 & - & 02 \\
\hline Total & 200 & 33 & 177 & 23 \\
\hline \hline
\end{tabular}

Área I - Área de Repouso da Fonte Alimentar (Poleiro)

Área II - Área de Alimentação das Aves

Área III - Barreira de Transposição entre o Local da Soltura e a Fonte de Alimentação (Tijolos Furados e Tela) Área IV - Área de Soltura das Ninfas

\section{CONCLUSÃO}

As informações obtidas com a realização do presente trabalho, permitem dizer que os exemplares do $4^{\circ}$ estádio de Triatoma brasiliensis, não alimentados, soltos na área IV do galinheiro experimental, buscaram em sua maioria, o alimento disponível (aves) e se alojaram e permaneceram nas imediações das fontes. Os dados alcançados nos mostram aquilo que era previsível e somente agora constatado através de observação experimental para a espécie. A importância do estímulo alimentar na atividade locomotora dos triatomíneos, já foi observada em Triatoma infestans por Mac Cord et al. (1983b). 
Além do interesse estritamente epidemiológico, o trabalho, supõe-se, apresenta também interesse para as atividades de controle dos vetores da doença de Chagas. Nesse aspecto, vale ressaltar suas implicações no referente à técnica da borrifação dos inseticidas, ou seja, a possibilidade de redução na área de aplicação. De uma aplicação em todas as paredes da casa ou anexo, sugerimos a aplicação focal, apenas nas proximidades das possíveis fontes alimentares presentes no mesmo, acrescido de que a adoção de expurgo focal torna-se menos oneroso e também um fator importante na redução da poluição ambiental.

\section{RESUMO}

RODRIGUES, V.L.C.C.; FERRAZ-FILHO, A. N.; ISHIHATA, G. K. \& SILVA, E. O. R. Triatoma brasiliensis (Neiva, 1911) (Hemiptera, Reduviidae): Observações Sobre seu Comportamento em Relaçãoà Fonte Alimentar em Galinheiro Experimental. Cad. Saúde Públ., Rio de Janeiro, 11 (2): 266-270, abr/jun, 1995.

O presente trabalho tem a finalidade de estudar o comportamento de triatomíneos no interior de ecótopos artificiais. Para tanto, construiu-se galinheiro experimental com paredes internas formadas por blocos de barro desmontáveis, abrigando aves como fonte alimentar. $\mathrm{O}$ galinheiro foi subdividido em 4 áreas, duas destas separadas por telas e tijolos furados, sendo a menor o local de soltura dos triatomíneos e a maior o galinheiro propriamente dito. Dessa maneira buscou-se a coleta de informações a respeito do deslocamento, sobrevida e locais de abrigo de ninfas do $4^{\circ}$ estádio de Triatoma brasiliensis, na certeza de que os mesmos iriam se acomodar nas proximidades da fonte alimentar. As paredes do galinheiro foram desmontadas e pesquisadas mensalmente durante 6 meses e as ninfas encontradas, marcadas com diferentes cores de acordo com o mês da leitura e a posição das mesmas nas áreas. Os resultados alcançados mostraram que $88,5 \%$ das ninfas estavam engorgitadas e foram encontradas próximas ao local de repouso (poleiro) da fonte alimentar. Após a primeira alimentação, não abandonavam as proximidades da fonte. Os resultados alcançados apresentam, não apenas interesse epidemiológico, como também interesse na área de controle dos vetores da doença de Chagas, tendo em vista as possíveis implicações quanto aos procedimentos na aplicação do inseticida.

Palavras-Chave: Triatoma brasiliensis; Triatominae; Doença de Chagas;

\section{REFERÊNCIAS BIBLIOGRÁFICAS}

ALENCAR, J. E., 1987. História Natural da Doença de Chagas no Estado do Ceará. Fortaleza: Imprensa Universitária da Universidade Federal do Ceará.

COSTA, M. J. \& PERONDINI, A. L. P., 1973 Resistência do Triatoma brasiliensis ao jejum Revista de Saúde Pública, 7: 207-217.

FORATTINI, O. P., 1980. Biogeografia origem e distribuição da domiciliação de triatomineos no Brasil. Revista de Saúde Pública, 14: 265-299.

LENT, H. \& WYGODZINSKY, P., 1979. Revision of the triatominal (Hemiptera, Reduviidae), and their significance as vectors of Chagas' disease. Bulletin of the American Museum of Natural History, 163: 206-208.

LUCENA, D. T., 1958. Epidemiologia da Doença de Chagas em Pernambuco. II nota sobre as espécies de triatomíneos. Revista Brasileira de Malariologia e Doenças Tropicais, 4: 355-368.

1960. Evolução do Triatoma brasiliensis (Neiva, 1911) no laboratório. Revista Brasileira de Biologia, 20: 295-302.

MAC CORD, J. R.; JUBERG, P. \& RAYMUNDO, J. S., 1983a. Técnica para observação do comportamento do Triatoma infestans (Klug, 1834) em uma miniatura de casa de pau-a-pique e sapê. Memórias do Instituto Osvaldo Cruz, 78: 153163.

MAC CORD, J. R.; JUBERG, P. \& LIMA, M. M., 1983b. Marcação individual de triatomíneos para estudos comportamentais e ecológicos. Memórias do Instituto Osvaldo Cruz, 78: 473-476. 